\title{
Les secrets de la salle de conseil
}

\section{Werner Bauer}

Dr méd., président de I'ISFM

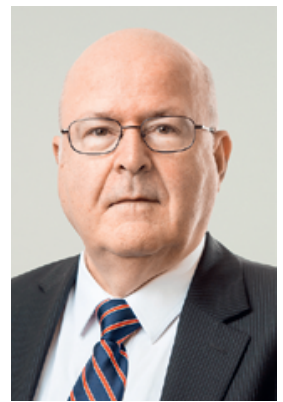

En feuilletant la presse des Grisons, mon regard s'est arrêté sur la rubrique "Aus der Ratsstube». Elle rapporte les décisions du Conseil communal. Le choix des termes m'a plu, car il donne au lecteur l'impression d'une salle de conseil chaleureuse, dans laquelle il pourrait se poster en observateur: je m'imagine une pièce décorée d'une élégante frisette en pin, avec des vitres en cul de bouteille.

La direction de l'Institut suisse pour la formation médicale postgraduée et continue tient elle aussi des réunions, dans une sobre salle de Berne, sans aucune boiserie. Beaucoup de lecteurs n'ont aucune idée des dossiers qui peuvent figurer à l'ordre du jour de l'ISFM. Je saisis l'opportunité de cette rubrique «Et encore» pour vous faire découvrir la "salle de conseil de l'ISFM», dans le pur respect de toutes les règles de confidentialité, naturellement.

Comme dans toutes les réunions, la séance démarre avec l'approbation du procès-verbal et le message du président. L'expérience montre que les membres des instances responsables du procès-verbal se divisent en deux groupes: le plus petit est celui qui demande la parole le plus régulièrement possible pendant cette phase d'approbation, réclamant une précision ou indiquant une coquille; l'autre groupe, plus important, a survolé le procès-verbal en arrivant dans la salle, voire ne l'a pas lu, étant sûr qu'il est exact, et signifie avec empressement son assentiment. Mais la direction de l'ISFM n'est composée que de lecteurs attentifs du procès-verbal, qui constituent un troisième groupe, autrement difficile à trouver.

Le message du président et du directeur n'est pas si anodin pour le déroulement de la séance, même s'il a tendance à être un peu long ou qu'il déclenche des commentaires inattendus. Si le message dure au-delà de la première heure, c'est que la présidence de la séance est sérieusement sollicitée.

Le point suivant de l'ordre du jour est le «développement du nouveau logbook». Il s'agit là de l'activité principale de l'ISFM, car toutes les exigences respectées et étapes de formation postgraduée passées sont documentées dans ce logbook, qui doit être rempli pour obtenir le titre de spécialiste. Le logbook actuel, qui ne répond plus aux exigences et ne peut plus être développé, doit être remplacé par une nouvelle version. Ce point de l'ordre du jour génère un sentiment mitigé de confiance et d'angoisse, car c'est la direction qui en assume la responsabilité, mais qu'elle dépend pour cela des experts informatiques. Nous nous sentons relativement compétents pour ce qui est des questions médicales et de la formation postgraduée et continue. Mais pour ce qui est du pilotage et de l'accompagnement des projets informatiques, il nous faut faire confiance aux professionnels et croiser les doigts.

Un autre point de l'ordre du jour nécessite aussi de faire confiance, à savoir le traitement et l'approbation de la révision des programmes de formation postgraduée. Il est intéressant de noter la fréquence à laquelle chacune des 45 sociétés qui gèrent les titres de spécialistes manifeste la volonté d'actualiser son programme de formation. Il peut s'agir d'ajouter ou de supprimer des objectifs pédagogiques, d'augmenter ou de diminuer le nombre d'opérations exigées ou de préciser les critères de reconnaissance des établissements de formation postgraduée. Ce type de décision pose parfois des problèmes, par exemple quand cela nécessite de lourdes dispositions transitoires administratives, ou quand la démarche est considérée comme hostile par une autre société de discipline médicale, qui craint que cela ne vienne empiéter sur les plates-bandes de ses propres objectifs pédagogiques. Une conciliation est alors nécessaire!

Après une pause pour prendre l'air et boire un café, une série d'autres points attendent d'être examinés. Il y a tout d'abord l'obligation les finances, puis il faut préparer les congrès, développer le projet «teach the teachers», destiné au soutien didactique de ceux qui assurent la formation postgraduée, parler de la reconnaissance des certificats des organismes européens et de leur intégration dans notre système de gestion des titres, aborder la délicate question de savoir si nous devons diffuser les adresses et autres données aux organisations partenaires, sous quelle forme, et si nous avons le droit de le faire. Certains points de l'ordre du jour pousseraient presque certains à quitter la salle, d'autres font grincer des dents.

Certains soupirent par exemple quand nous devons rconnaître que la formation postgraduée doit servir d'écran à des intérêts politiques ou lorsqu'une autorité laisse libre cours à son imagination et soumet des propositions bureaucratiques et réglementaires difficilement compréhensibles.

Enfin est abordé le point «Divers», avant que les lumières de la salle de conseil ne s'éteignent. 